\title{
Chikungunya: Facts and Responsibilities to Combat
}

\author{
*MM Rahman ${ }^{1}$, MMR Siddiqui ${ }^{2}$
}

\section{Introduction}

Chikungunya is a viral disease transmitted to humans by infected female Aedes mosquitoes. It is characterized by an abrupt onset of fever accompanied by joint pain with common signs and symptoms that includes muscle pain, headache, nausea, fatigue and rash. Thus the virus can cause acute, sub-acute or chronic disease. Most patients recover fully, but in some cases joint pain may persist for weeks, several months, or even years. Occasional cases of eye, neurological and heart complications have been reported, as well as gastrointestinal complaints. Complications are not common, but in older people, the disease may lead to the cause of death. The symptoms in infected individuals are mild and the infection may go unrecognized, or be misdiagnosed in areas where dengue occurs. The disease shares some clinical signs with dengue and zika, and can be misdiagnosed in areas where those are common. The treatment is focused on relieving the symptoms only with analgesic, antipyretic, antihistamine, optimal fluids and by bed rest. There is no commercial chikungunya vaccine.

Chikungunya is a mosquito-borne viral disease first described during an outbreak in southern Tanzania in 1952. It is an RNA virus that belongs to the alphavirus genus of the family Togaviridae. The name "chikungunya" derives from a word in the Kimakonde language, meaning "to become contorted", and describes the stooped appearance of sufferers with joint pain (arthralgia). The proximity of mosquito breeding sites to human habitation is a major significant risk factor for chikungunya. The disease mostly occurs in
Africa, Asia and the Indian subcontinent. However a major outbreak in 2015 affected several countries of the Region of the Americas as well. The name Chikungunya comes from word "swahili" meaning 'that which bends up' referring to the stooped posture the victim adopt to relief the joint pain. Immunity is long lasting.

Commonly the mosquitoes involved in the transmission of disease are Aedes aegypti and Aedes albopictus, those two species are also transmit other mosquito-borne viruses, including dengue. Ae. aegypti is more closely associated with human habitation and uses indoor breeding sites, including flower vases, water storage vessels and concrete water tanks in bathrooms, as well as the same artificial outdoor habitats as Ae. albopictus. Both species are found biting outdoors, but Ae. aegypti also feed indoors. The species Ae. albopictus thrives in a wider range of waterfilled breeding sites than Ae. aegypti, including coconut husks, cocoa pods, bamboo stumps, tree holes and rock pools, in addition to artificial containers such as vehicle tyres and saucers beneath plant pots. This diversity of habitats explains the abundance of Ae. albopictus in rural as well as peri-urban areas and shady city parks. After the bite of an infected mosquito, onset of illness occurs usually between 4 and 8 days but it can range from 2 to 12 days. The spread of Chikungunya virus carrying mosquitoes has increased due to the lack of cleanliness and the almost closure of mosquito elimination program in the city. The incidence of Chikungunya increases after heavy rains due to number of increased breeding places the vector prefers.

${ }^{1}$ Prof. Dr. Md. Mahfuzar Rahman, Department of Community Medicine, AKMMC

${ }^{2}$ Dr. Md. Mahmudur Rahman Siddiqui, Associate Professor, Department of Medicine, AKMMC

*Corresponding Author

AKMMC J 2017; 8(2) : 83-84 
Diagnostic method can be serological tests, such as enzyme-linked immunosorbent assays (ELISA), confirm the presence of IgM and IgG anti-chikungunya antibodies. IgM antibody levels are highest 5 days after the onset of illness and persist for about 2 months. Samples collected during the first week after the onset of symptoms can be tested by virological methods (RT-PCR).

The vector breeding sites to human habitation is a significant risk factor for chikungunya as well as for other diseases that these species transmit. Prevention and control mostly depends on reducing the number of natural and artificial water-filled containers that support breeding of mosquitoes. It requires community mobilization. During outbreaks, insecticides may be sprayed to kill flying mosquitoes, and to treat water in containers to kill the immature larvae. To deal with protection during outbreaks of chikungunya, clothing which minimizes skin exposure to the day-biting vectors is advised. Repellents can be applied to exposed skin or to clothing in strict accordance with product label instructions. World
Health Organization (WHO) encourages countries to develop and maintain the capacity to detect and confirm cases, manage patients and implement social communication strategies to reduce the presence of the mosquito vectors. Therefore it is essential to enhance awareness on individual protection against Ae. mosquito bite in addition to social responsibility regarding elimination of breeding places towards effective control to combat this disease in particular.

\section{References}

1. Surayakantha, A.H; Community Medicine with recent advances, $3^{\text {rd }}$ edition (New Delhi, India, Japee Brothers Medical Publishers (p) Ltd, 2014 section-5), $452-453$

2. http:// dailyasianage.com/ news/62036/ chikungunyaspreading-first

3. http:// www.who.int/mediacentre/ factsheets/fs327/en/

4. http://www.chikungunyavirusnet.com/ guidelines/42who-chikungunya-guidelines-and-documents.html

5. https:// www. youtube.com/ watch?v $=\mathrm{d} 6 \mathrm{rVte} 7 \mathrm{~h}$ Ovc 